\title{
Urinary Exosomal MicroRNA Profiling in Incipient Type 2 Diabetic Kidney Disease
}

\author{
Yijun Xie, Yijie Jia, Xie Cuihua, Fang Hu, Meng Xue, and Yaoming Xue \\ Department of Endocrinology and Metabolism, Nanfang Hospital, Southern Medical University, Guangzhou, \\ Guangdong 510515, China \\ Correspondence should be addressed to Yaoming Xue; xueyaoming999@126.com
}

Received 2 May 2017; Revised 19 June 2017; Accepted 28 June 2017; Published 5 September 2017

Academic Editor: Ahmed A. Elmarakby

Copyright (C) 2017 Yijun Xie et al. This is an open access article distributed under the Creative Commons Attribution License, which permits unrestricted use, distribution, and reproduction in any medium, provided the original work is properly cited.

Background. Albuminuria is an early sign but not a strong predictor of diabetic kidney disease (DKD). Owing to their high stability, urinary exosomal miRNAs can be useful predictors of the progression of early-stage DKD to renal failure; fluid biopsies are ideal for detecting abnormalities in these miRNAs. The aim of this study was to identify novel differentially expressed miRNAs as urine biomarkers for type $2 \mathrm{DKD}$ by comparing between patients of type 2 diabetes (T2D) with and without macroalbuminuria. Methods. Ten patients with T2D, including five who had no renal disease and five with macroalbuminuria (DKD G1-2A3), were selected for this study. Exosome- (UExo-) derived miRNA profiles were used to identify candidate biomarkers, a subset of which was verified using quantitative reverse transcription PCR. Results. A total of 496 UExo-derived miRNA species were found to be differentially expressed ( $>2$-fold) in patients with $\mathrm{DKD}$, compared to those with T2D. A validation analysis revealed that three miRNAs (miR-362-3p, miR-877-3p, and miR-150-5p) were upregulated and one (miR-15a-5p) was downregulated. These miRNAs might regulate DKD through p53, mTOR, and AMPK pathways. Conclusions. In conclusion, UExo-derived miRNAs were altered in type 2 DKD. MiR-362-3p, miR-877-3p, miR-150-5p, and miR-15a-5p might be novel biomarkers for incipient DKD.

\section{Introduction}

Diabetic kidney disease (DKD) is a type of chronic kidney disease $(\mathrm{CKD})$ caused by diabetes mellitus. It is the leading cause of end-stage renal diseases in Western countries [1] and has been reported in more than $30 \%$ of patients with type 2 diabetes mellitus (T2DM) in China [2]. DKD has an insidious onset; once proteinuria occurs, progression to end-stage renal disease is rapid. Microalbuminuria does not accurately predict DKD; [3] new biomarkers to identify the early stage of DKD are therefore urgently needed.

MicroRNAs (miRNAs) are a group of short $(\sim 22 \mathrm{nt})$, small, noncoding RNAs that posttranscriptionally regulate gene expression by suppressing target mRNAs [4]. Previous experimental studies have suggested the involvement of miRNAs with the pathogenesis of renal diseases $[5,6]$ and the development of DKD [7]. Cell-free circulating miRNAs are known to be stable in a variety of body fluids, including urine.
Urine is a suitable source of biomarkers for kidney diseases, and several urinary miRNA biomarkers have been identified for IgA nephropathy [8], nephrotic syndrome [9], lupus nephritis [10], and DKD in type 1 diabetes mellitus [11].

Exosomes $(40-100 \mathrm{~nm})$ are cup-shaped vesicles derived from the cellular endocytic compartment that can be isolated from urine and other body fluids such as serum, plasma, saliva, and milk [12]. Because exosomes can carry proteins, nucleotides, deoxynucleotides, and miRNAs to distant target cells, they represent an important mechanism for cell-to-cell communication [13]. Urinary exosome- (UExo-) derived miRNAs may be better diagnostic markers than free miRNAs. UExo-derived miRNAs are protected from endogenous RNase activity, are remarkably stable, and are not easily confounded by plasma miRNAs that pass the glomerular filtration barrier [14]. Changes in UExo-derived miRNAs have been found to be significantly correlated with the 
progression of focal segmental glomerulosclerosis [15] and DKD in type 1 diabetes mellitus [16].

In vitro studies and analyses of the urinary exosomes of patients with T2DM have shown that increased levels of miR-192 and miR-215 promote renal injury in DKD [17]. It is to be noted that neither free urinary miRNA profiling in patients with type I DKD [18] nor UExo-derived miRNA profiling in patients with T1 [16] and T2 [19] DKD has been able to verify the association between miR-192 and DKD.

Our previous studies [20] have shown that the expression of UExo-derived miR-192 increased in patients with T2DM with microalbuminuria but decreased in those with macroalbuminuria. The combined analysis of the expression levels of UExo-derived miR-192 and TGF- $\beta 1$ might provide new insights into the pathology of incipient $\mathrm{DKD}$. The underlying mechanism of the association between UExo-derived miRNAs and DKD pathogenesis deserves exploration.

Circulating levels of total and specific miRNAs are known to be lower in patients with severe chronic renal failure than in those with mild renal impairment or normal renal function [14]. We therefore assumed that the initially upregulated UExo-derived miRNAs in patients with renal failure would be downregulated.

It is important to consider kidney function when identifying miRNAs as biomarkers of DKD. Previous analyses of the miRNA signature in urinary exosomes of patients with type 2 DKD [19] have focused on the decline in glomerular filtration rate (GFR). Differences in UExo-derived miRNAs between patients with normal or mildly decreased eGFR (G1 or G2) and those with severely increased albuminuria (A3) [21] are unclear. Here, we aimed to identify novel miRNA candidates that reflected changes in incipient type $2 \mathrm{DKD}$; these miRNAs could have implications in preventing or delaying disease progression.

\section{Materials and Methods}

2.1. Patient Characteristics. Ten patients, who were diagnosed with T2DM and admitted to the Department of Endocrinology and Metabolism, Nanfang Hospital, Southern Medical University, Guangdong, China, from January to November 2016, were enrolled for the study. The exclusion criteria were as follows: type 1 diabetes mellitus; nondiabetic kidney disease; acute diabetic complications (diabetic ketoacidosis, hyperosmolar nonketotic coma, and inflammatory disorders); diabetes mellitus complicated with severe heart, liver, or renal insufficiency (uremia); neoplastic disorders; urinary tract infection; rheumatic diseases; use of nephrotoxic drugs; hepatitis B; pregnancy; stroke; and occlusive peripheral vascular disease. Patients with an eGFR $<60 \mathrm{~mL} / \mathrm{min} / 1.73 \mathrm{~m}^{2}$ and those with macroalbuminuria or microalbuminuria who did not present with diabetic retinopathy were also excluded.

Urine samples were obtained from each patient. For the miRNA array, patients were classified into two groups based on the degree of albuminuria, a normoalbuminuria group (urine albumin-to-creatinine ratio (ACR) $<2.5 \mathrm{mg} / \mathrm{mmol}$ and urinary albumin excretion rate (AER) $<30 \mathrm{mg} / 24 \mathrm{~h}$, $N=5$ ) and a macroalbuminuria group (ACR $>25 \mathrm{mg} / \mathrm{mmol}$ or AER $=300-800 \mathrm{mg} / 24 \mathrm{~h}, N=5$ ).
Thirty additional patients with T2DM were enrolled for verification using $\mathrm{qRT}-\mathrm{PCR}$. The exclusion criteria were the same as those described previously, but only patients with eGFR $<60 \mathrm{~mL} / \mathrm{min} / 1.73 \mathrm{~m}^{2}$ were selected. Thus, 40 patients were classified into two groups, a normoalbuminuria group (ACR $<2.5 \mathrm{mg} / \mathrm{mmol}$ and $A E R<30 \mathrm{mg} / 24 \mathrm{~h}$, $N=20$ ) and a macroalbuminuria group (ACR $>25 \mathrm{mg}$ / mmol or AER $>300 \mathrm{~g} / 24 \mathrm{~h}, N=20$ ).

This study was approved by the ethics committee of Nanfang Hospital, Southern Medical University. All patients provided written informed consent prior to participating in the study.

2.2. Urinary Exosomal Isolation. Initially, $100 \mathrm{~mL}$ of firstmorning urine was collected from all subjects in sterile containers. All the samples were processed within $1 \mathrm{~h}$ of collection. Urinary cells were removed by centrifugation at $2000 \times g$ (Rotor: JA-20; Beckman Coulter, Fullerton, CA, USA) for $15 \mathrm{~min}$ at $4^{\circ} \mathrm{C}$ and at $10,000 \times g$ for $15 \mathrm{~min}$ at $4^{\circ} \mathrm{C}$. Subsequently, the supernatant was ultracentrifuged at $170,000 \times g$ for $70 \mathrm{~min}$ at $4^{\circ} \mathrm{C}$ (Rotor: SW 32Ti; Beckman Coulter, Brea, CA, USA). After removing the supernatant, the pellets were washed with $8 \mathrm{~mL}$ of sterile phosphate-buffered saline and ultracentrifuged at $170,000 \times g$ for $70 \mathrm{~min}$. The pellets were then suspended in $100 \mu \mathrm{L}$ of phosphate-buffered saline and stored at $-80^{\circ} \mathrm{C}$ for further analyses.

2.3. Total RNA Extraction. The UExo samples were treated with $0.1 \mu \mathrm{g} / \mu \mathrm{L}$ RNase A (BioSharp, USA) for $10 \mathrm{~min}$ at $37^{\circ} \mathrm{C}$. Total RNA was isolated using TRIzol (Invitrogen/ DingGuo, Beijing, China) and the miRNeasy Mini Kit (QIAGEN, Hilden, Germany), according to the manufacturer's instructions. The extracted RNA was quantified and assessed using a NanoDrop ND-1000 Spectrophotometer (Thermo Fisher Scientific, Wilmington, DE, USA). Quality control was performed using the Agilent 2100 Bioanalyzer.

2.4. miRNA Profiling and Data Analysis. After quality control, the miRCURY ${ }^{\mathrm{TM}} \mathrm{Hy}{ }^{\mathrm{TM}} / \mathrm{Hy}^{\mathrm{TM}}$ Power Labeling Kit (Exiqon, Vedbaek, Denmark) was used to make a $25 \mu \mathrm{L}$ mixture containing $1 \mu \mathrm{L}$ of RNA, according to the manufacturer's guidelines for miRNA labeling. After labeling, the Hy3-labeled samples were hybridized on the miRCURY LNA Array (v.19.0) (Exiqon). Finally, the slides were scanned using the Axon GenePix 4000B Microarray Scanner (Axon Instruments, Foster City, CA, USA). The scanned images were then imported into GenePix Pro 6.0 software (Axon) for grid alignment and data extraction. After normalization, miRNAs that showed significant differential expression between the two groups were identified based on fold changes $>2$ and $P$ values $<0.05$. Hierarchical clustering was performed to identify differences in the miRNA expression profiles among samples.

2.5. Animals and Treatment. Six male C57BL/6J mice (3-4week old, weighing 15-16g) were obtained from the Animal Center of Guangdong province. After four weeks on highfat diets, the mice received a single injection of STZ $(120 \mathrm{mg} / \mathrm{kg}$, i.p., in citrate buffer, $\mathrm{pH}=4.5, \mathrm{MP}$ Biomedicals $)$. Blood glucose was measured weekly and was found to have 
TABLE 1: Clinical and laboratory parameters of patients with type 2 diabetes.

\begin{tabular}{lcc}
\hline & Normoalbuminuric DM2 & Macroalbuminuric DM2 \\
\hline$N$ & 5 & 5 \\
Age (years) & $53.40 \pm 3.90$ & $54.60 \pm 2.98$ \\
Gender (male/female) & $3 / 2$ & $3 / 2$ \\
Diabetes duration (years) & $8.00 \pm 1.58$ & $9.20 \pm 1.66$ \\
SBP $(\mathrm{mmHg})$ & $116.80 \pm 4.88$ & $118.40 \pm 3.70$ \\
DBP $(\mathrm{mmHg})$ & $76.20 \pm 3.07$ & $76.60 \pm 3.31$ \\
HbA1C $(\%)$ & $8.58 \pm 0.76$ & $9.20 \pm 0.92$ \\
Body mass index $\left(\mathrm{kg} / \mathrm{m}^{2}\right)$ & $26.18 \pm 1.42$ & $25.75 \pm 2.45$ \\
Serum creatinine $(\mu \mathrm{mol} / \mathrm{L})$ & $66.4 \pm 9.17$ & $66.60 \pm 7.00$ \\
eGFR $\left(\mathrm{mL} / \mathrm{min} \times 1.73 \mathrm{~m}^{2}\right)$ & $99.78 \pm 8.41$ & $100.60 \pm 5.38$ \\
Triglycerides $(\mathrm{mmol} / \mathrm{L})$ & $2.10 \pm 0.26$ & $1.53 \pm 0.27$ \\
Cholesterol $(\mathrm{mmol} / \mathrm{L})$ & $5.08 \pm 0.36$ & $4.78 \pm 0.42$ \\
LDL-C $(\mathrm{mmol} / \mathrm{L})$ & $3.32 \pm 0.29$ & $3.09 \pm 0.22$ \\
HDL-C $(\mathrm{mmol} / \mathrm{L})$ & $0.92 \pm 0.58$ & $1.03 \pm 0.15$ \\
Retinopathy $(\mathrm{y} / \mathrm{n})$ & $2 / 3$ & $5 / 0$ \\
ACR $(\mathrm{mg} / \mathrm{mmol})$ & $0.92 \pm 0.35$ & $30.90 \pm 3.92^{*}$ \\
AER $(\mathrm{mg} / 24 \mathrm{~h})$ & $11.60 \pm 2.52$ & $402.60 \pm 24.738^{*}$ \\
\hline
\end{tabular}

Data are shown as mean \pm SEM; DM2: type 2 diabetic patients; SBP: systolic blood pressure; DBP: diastolic blood pressure; HbA1C: glycosylated hemoglobin; GFR: glomerular filtration rate; LDL-C: low-density lipoprotein cholesterol; HDL-C: high-density lipoprotein cholesterol; ACR: albumin/creatinine ratio; AER: albumin excretion rate; ${ }^{*} P<0.001$, macroalbuminuric versus normoalbuminuric patients.

reached a sustained level of $>16.7 \mathrm{mM}$ at 16 weeks, which was considered an indicator of hyperglycemia. The mice in the DM group were sacrificed after modeling $(N=3)$; twelve weeks later, the mice of the DKD group were also sacrificed $(N=3)$. Renal cortex samples were snap frozen in liquid nitrogen and stored at $-80^{\circ} \mathrm{C}$ for analysis. The protocols for all animal studies conformed to the established institutional and state guidelines for the care and use of laboratory animals. Total RNA was obtained from renal cortex tissues using the TRIzol reagent, as described previously.

2.6. Quantitative Reverse Transcription PCR. The miRNAs were reverse transcribed to complementary DNA (cDNA) using the miRcute miRNA First-strand cDNA Synthesis Kit (TIANGEN, Beijing, China). RT-PCR was performed using the miRcute miRNA qPCR Detection Kit (TIANGEN) and the LightCycler480 Real-Time PCR System (Roche; Hoffmann-La Roche Ltd., Basel, Switzerland). The relative expression levels of the target miRNAs were calculated by the comparative $2^{-\Delta \Delta C T}$ method with U6 snRNA as the internal control. Primers for miR-362-3p, miR-877-3p, miR-150$5 p$, miR-15a-5p, and U6 were obtained from TIANGEN.

\subsection{Bioinformatics Analysis of miRNA Target Genes and Pathways}

2.7.1. Identification of miRNA Target Genes. To infer the putative targets of differentially expressed miRNAs, two algorithms were used: targetScan7.1 (http://www.targetscan.org/ vert_71) and mirdbV5 (http://mirdb.org/miRDB). Genes for which consistent results were obtained using both databases were considered target genes of the miRNAs. An interaction network of differentially expressed miRNAs and predicted target genes was constructed using Cytoscape v2.8.3 (http://cytoscape.org).

2.7.2. Functional Enrichment Analysis. To investigate the biological functions of the target genes, a Gene Ontology (GO) function and Kyoto Encyclopedia of Genes and Genomes (KEGG) pathway enrichment analyses were performed using the DAVID tool (https://david.ncifcrf.gov/). $P<0.05$ and FDR $<0.05$ was considered statistically significant.

2.8. Statistical Analyses. All the data were analyzed using the SPSS 20.0 software. All results are presented as mean \pm SEM. The results of the qRT-PCR were analyzed using Student's $t$-tests. CT values above 45 were considered undetectable, and $P<0.05$ was considered significant.

\section{Results}

3.1. Patient Characteristics. Clinical and laboratory characteristics of the participants are summarized in Table 1 . No significant differences were observed between the normoand macroalbuminuric groups in age, gender, SBP, DBP, BMI, serum creatinine, eGFR, triglycerides, cholesterol, LDL-C, and HDL-C. Although the duration of diabetes and the HbAlc values were greater in the macroalbuminuric patients than in the normoalbuminuric ones, these differences were not statistically significant. As expected, the ACR $(t=7.619, P<0.001)$ and AER $(t=15.724, P<0.001)$ values were higher in patients with macroalbuminuria than in those with normoalbuminuria. 


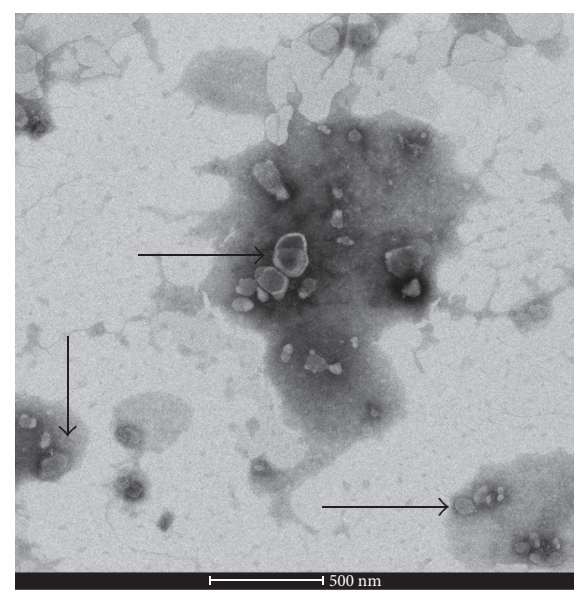

(a)

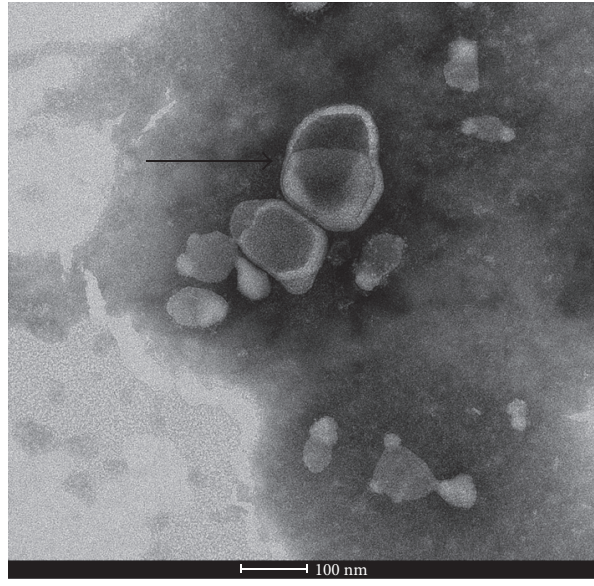

(b)

FIgURE 1: Identification of urinary exosomes by transmission electron microscopy.

3.2. Urinary Exosome Characteristics. Vesicles isolated from the urine collected overnight were less than $100 \mathrm{~nm}$ in size, with a characteristic cup-shaped morphology, as observed by electron microscopy (Figure 1). The total RNA extracted from exosomes was analyzed using an Agilent 2100 Bioanalyzer, and the urinary exosomes were found to be rich in miRNAs (Figure S1 in Supplementary Material available online at https://doi.org/10.1155/2017/6978984).

3.3. Differentially Regulated UExo-Derived miRNAs in Patients with Type 2 DKD. In total, 3100 miRNAs were detected in the urinary exosomes of the macro- and normoalbuminuric patients with T2DM. Differential miRNA profiling revealed 496 miRNAs that were differentially expressed at least 2 -fold, in matched pairs of macro- and normoalbuminuric patients, including 203 upregulated miRNAs and 188 downregulated ones (Table 2, Figure 2). For biological analysis, we selected two miRNAs that showed the most significant differential expression, hsa-miR-362-3p and hsa-miR-3191$5 \mathrm{p}$. We also selected two miRNAs that were differentially expressed in both CKD [22] and DKD, hsa-miR-877-3p and hsa-miR-15a-5p. Based on the promoter-binding region upstream of the miRNAs, six miRNAs associated with the NF- $\kappa$ B pathway were selected, namely, hsa-miR-150-5p, hsa-miR-186-5p, hsa-miR-491-5p, hsa-miR-133b, hsa-miR638, and hsa-miR-324-3p. MiR-362-3p is known to be upregulated in human kidney allografts with tubulointerstitial fibrosis [23]. Previous studies have shown an association between miR-150-5p and proteinuria and eGFR decline [24]. To validate the differentially expressed miRNAs, qRT-PCR was performed for miR-362-3p, miR-877-3p, miR-15a-5p, and miR-150-5p.

3.4. Quantitative Reverse Transcription PCR. A total of 20 patients with T2DM and 20 patients with DKD were recruited to confirm the results obtained for the screening cohort. The expression levels of UExo-derived miR-362-3p, miR-877-3p, miR-150-5p, and miR-15a-5p were analyzed
TABLE 2: Differentially expressed miRNAs.

\begin{tabular}{lcc}
\hline Name & Fold change (DKD versus DM) & $P$ value \\
\hline hsa-miR-362-3p & 21.88235 & 0.001172371 \\
hsa-miR-877-3p & 4.261229 & $9.5308 e-08$ \\
hsa-miR-150-5p & 3.477725 & 0.000107498 \\
hsa-miR-491-5p & 2.29545 & 0.031921743 \\
hsa-miR-133b & 2.262081 & 0.003187535 \\
hsa-miR-638 & 2.186886 & $5.58287 e-06$ \\
hsa-miR-186-5p & 2.144811 & 0.00043897 \\
hsa-miR-324-3p & 2.061286 & 0.004536979 \\
hsa-miR-15a-5p & 0.373129 & 0.011076438 \\
hsa-miR-3191-5p & 0.003389 & $2.57342 e-07$ \\
\hline
\end{tabular}

DKD: diabetic kidney disease; DM: diabetic patients.

by qRT-PCR. The expression of miR-877-3p was found to be significantly upregulated in the UExos from patients with DKD, compared to those with T2DM, whereas the expression levels of miR-362-3p, miR-150-5p, and miR$15 a-5 p$ were not significantly different in the different groups (Figure 3). We then detected the differentially expressed UExo-derived miR-877-3p in the renal cortex of diabetic mice. The mice with DKD showed higher levels of urinary microalbumin and had higher renal weight index, than those in the DM group (Hu et al. unpublished data). As expected, miR-877-3p was upregulated in diabetic kidneys, but not in a statistically significant manner $(P=0.056$, Figure 4$)$. Overall, the qRT-PCR and miRNA array results were consistent, indicating that the miRNA array was reliable.

3.5. Target Gene Detection and Functional GO and Pathway Enrichment Analyses. A total of 724 target genes were predicted by both targetScan7.1 and mirdbV5 for the 10 selected miRNAs. An interaction network was constructed using Cytoscape (Figure S2). We performed a GO analysis for the target genes of the 10 miRNAs. Target genes of 


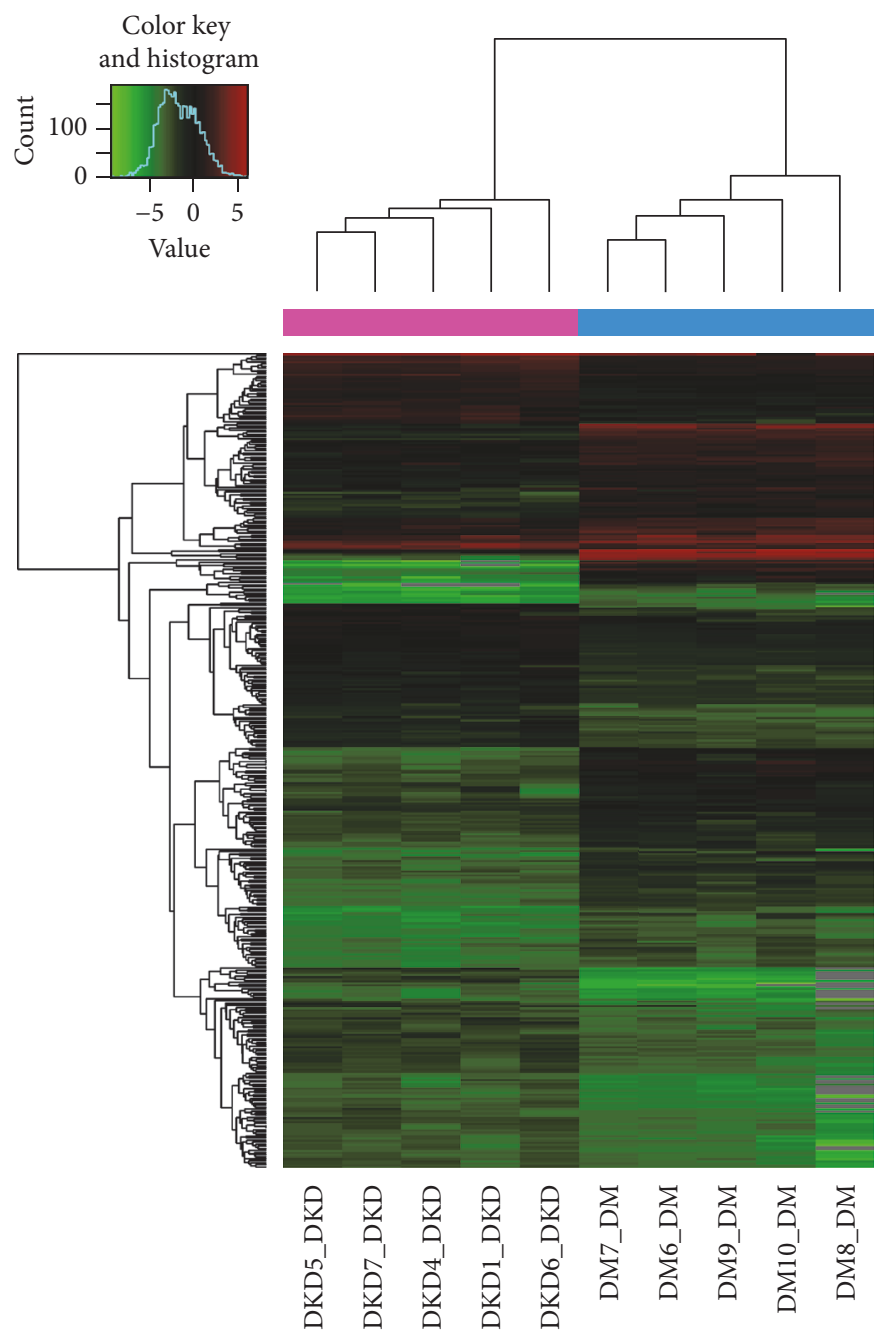

Figure 2: Clustering analysis of miRNA expression in urinary exosomes (DKD versus DM).

miRNAs in three major categories, biological process (BP), cellular component (CC), and molecular function (MF), were analyzed. In the BP category, significant enrichment of target genes was detected for macromolecular modification (GO: 0019222, $P=3.90 e-09$, count: 292$)$ and regulation of metabolic processes (GO: 0019222, $P=3.36 e-09$, count: 210 ) (Figure 5(a)). Membrane-bounded organelles (GO: 0043227, $P=9.55 e-08$, count: 488) and intracellular membranebounded organelles (GO: 0043229, $P=2.21 e-07$, count: 482 ) were the most relevant groups in the CC category (Figure 5(b)). Binding (GO: 0005488, $P=5.46523 e-08$, count: 547) and protein binding (GO: 0005515, $P=2.31 e-06$, count: 391$)$ in the MF category were the most significantly enriched terms associated with the target genes (Figure 5(c)).

We also performed a KEGG pathway enrichment analysis for differentially expressed miRNA target genes, with FDR $<0.05$ as the criterion for pathway detection. The target genes showed enrichment for 54 pathways, particularly pathways associated with malignant tumors, p53, neurotrophic factors, mTOR, AMPK, and other signaling pathways. The most highly enriched pathways were the
mTOR signaling pathway $(F D R=0.01717889)$ and the signaling pathways that regulate the pluripotency of stem cells $(F D R=0.01792762)($ Figures 5(d) and 6).

\section{Discussion}

Consistent with the results obtained here, UExos do indeed contain large amounts of miRNAs. We extracted UExos using a two-step differential ultracentrifugation process, which is considered ideal for exosome isolation from body fluids [25]. The size and features of the exosomes were determined by electron microscopy. We detected differences in UExo-derived miRNA profiles between patients with T2DM with and without macroalbuminuria, including 203 upregulated miRNAs and 188 downregulated ones, suggesting that UExo-derived miRNA signatures could be used as noninvasive biomarkers for predicting and diagnosing DKD.

Previous studies have explored UExo-derived miRNA profiles in patients with T2DKD with decreased renal functions. Albuminuria is apparent in the early stages of $\mathrm{DKD}$, years before renal failure; however, miRNA profiles 

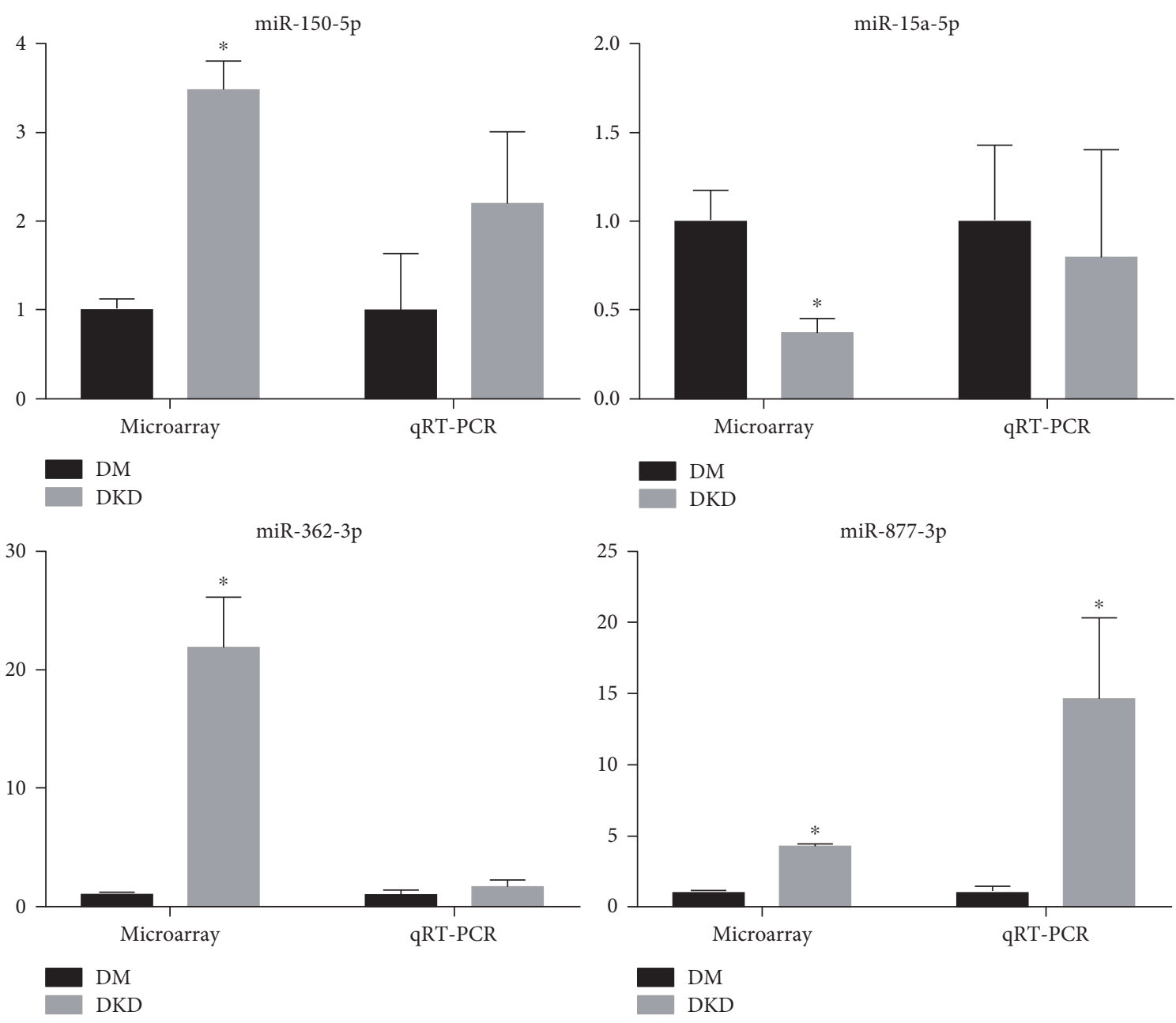

Figure 3: Fold changes in the expression levels of UExo-derived miRNAs. Significant differences against the DM and DKD groups are indicated by ${ }^{*} P<0.05$.

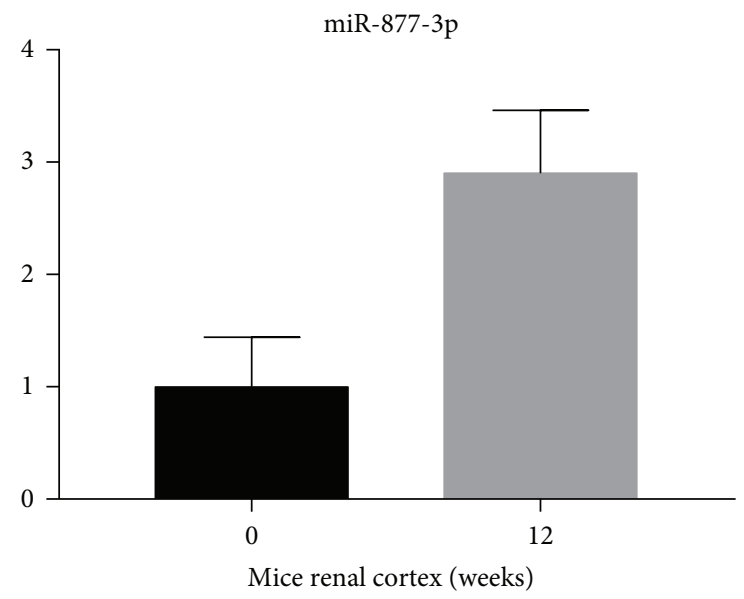

0 weeks

12 weeks

Figure 4: Expression levels of miR-877-3p in mice renal cortex.

at this early stage have not been investigated. Long-term observations have revealed that microalbuminuria is reversed in one-third of the patients after a decade and less than half the patients develop macroalbuminuria; this is more pronounced in patients with T2DM [26]. Initially elevated miRNA levels are eventually reduced during severe renal failure. In this study, we examined patients with T2DKD (G1-2A3), paving the way for developing methods to diagnose incipient T2DKD.

UExo-derived miRNA profiles of patients with CKD showed that the expression of $\mathrm{miR}-877-3 p$ was increased and that of miR-15a-5p was decreased [22]. In addition, the expression of miR-877-3p was markedly upregulated in interstitial pulmonary fibrosis [27], and circulating miR-15a-5p was negatively correlated with blood glucose levels [28]. Although there is no evidence for the association of these miRNAs with DKD, the findings in this study suggested that miR-877-3p and miR-15a-5p mediate fibrosis in DKD and are differentially expressed in patients with DKD and DM. These miRNAs might be useful for characterizing the similarities and differences between CKD and DKD; additional studies are needed to distinguish DKD from unexplained CKD.

The role of miRNAs in CKD progression has been studied extensively. Muralidharan et al. had found that the 


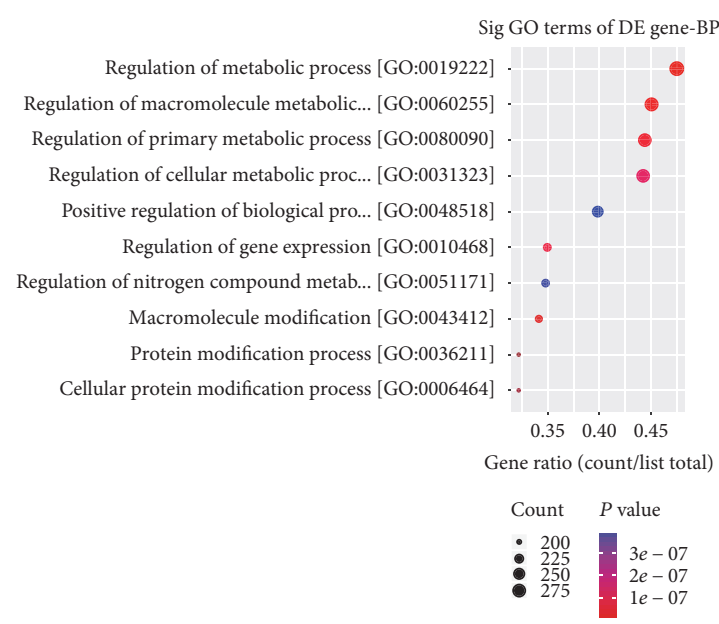

(a)

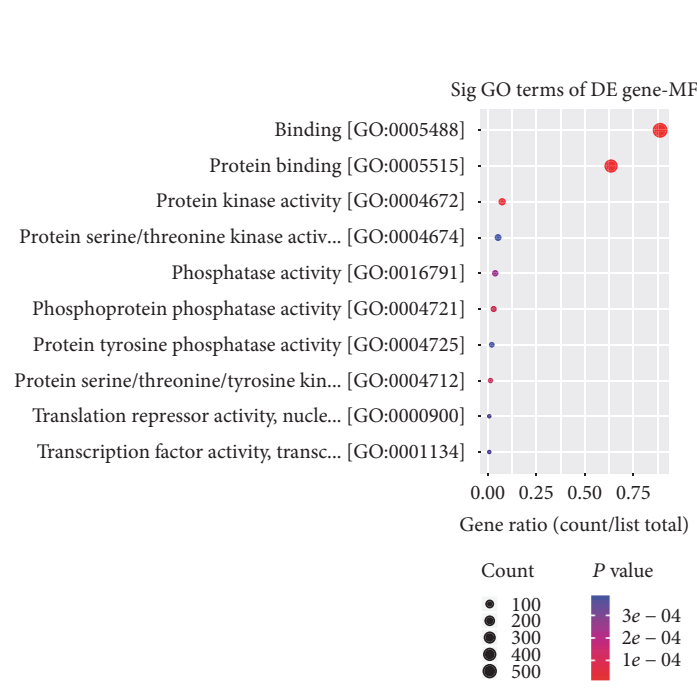

(c)

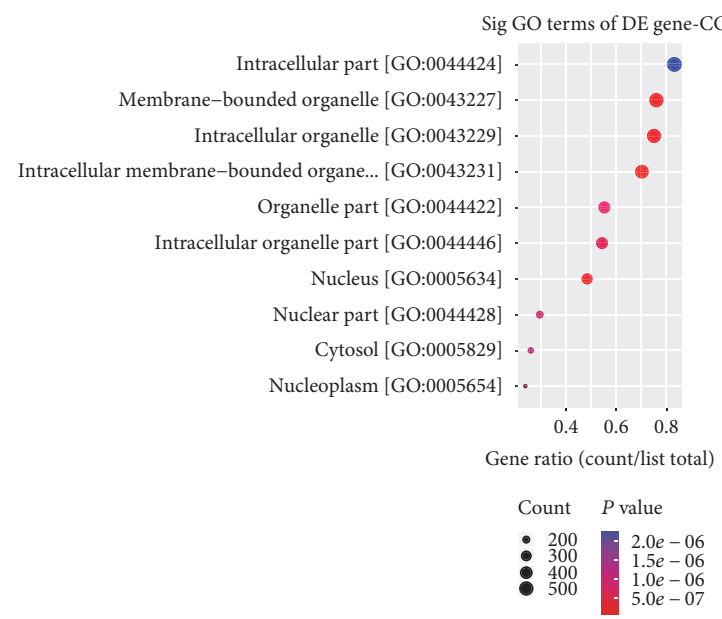

(b)

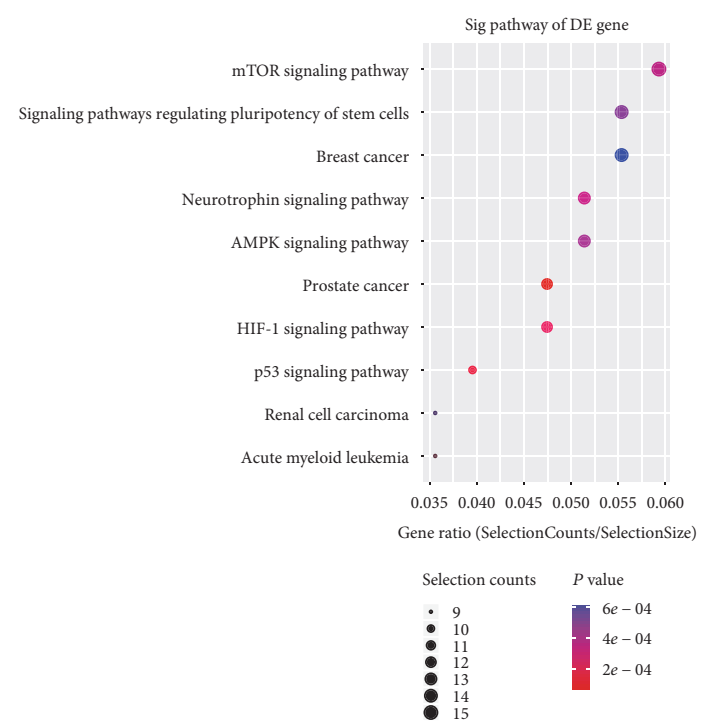

(d)

FIgURE 5: Gene ratio dot plot.

expression levels of 384 miRNAs from urine and 266 circulating miRNAs differed significantly between patients with CKD with eGFR $<30 \mathrm{~mL} / \mathrm{min} / 1.73 \mathrm{~m}^{2}$ and those with eGFR $\geq 30 \mathrm{~mL} / \mathrm{min} / 1.73 \mathrm{~m}^{2}$ [29]. Some miRNAs that are involved in CKD also have similar regulatory roles in DKD. In TGF- $\beta 1$-induced NRK52E cells, the expression of let-7b is decreased, while the activity of Smad3 is enhanced [30]. These changes have been observed both in early and advanced DKD mice and in a nondiabetic renal fibrosis model [31]. One factor that promotes kidney fibrosis is miR-21, which is distributed in the cortical glomeruli and renal tubular cells; it modulates the expression of matrix metalloproteinase9 (tissue metalloproteinase-1, MMP9/TIMP1). Additionally, miR-21 negatively regulates Smad7 to promote kidney damage [32] and plays a role in promoting fibrosis via multiple pathways in nondiabetic nephropathies such as IgA nephropathy $[33,34]$.

Unfortunately, patients with DKD rarely undergo renal biopsies, and so, we do not often detect differentially expressed UExo-derived miRNAs in diabetic kidneys.
Previous studies have shown that glomerular changes were attenuated ten weeks after modeling, with improved albuminuria [35]. We found that the level of miR-877-3p also increased in mice renal cortex. MiR-362-3p is known to be upregulated in human kidney allografts with tubulointerstitial fibrosis [23]. MiR-150 promotes the aging of renal glomerular mesangial cells and renal fibrosis [36]. The levels of UExo-derived miRNAs may be differentially altered in kidneys, and urinary exosomes could transport miRNAs to distant target cells. These differentially expressed UExoderived miRNAs could have two fates: they are either discharged or elevated in the kidney. Our results indicated that miR-877-3p belonged to the latter group, which is identical in kidney and urine samples.

We also compared the miRNAs identified in this experiment with those previously reported to be associated with DKD. Both miR-638 [19] and miR-133b [37] have been shown to be upregulated in urinary exosomes in patients with T2DKD. Consistent with these previous findings, we detected the differential expression of these miRNAs as well. 

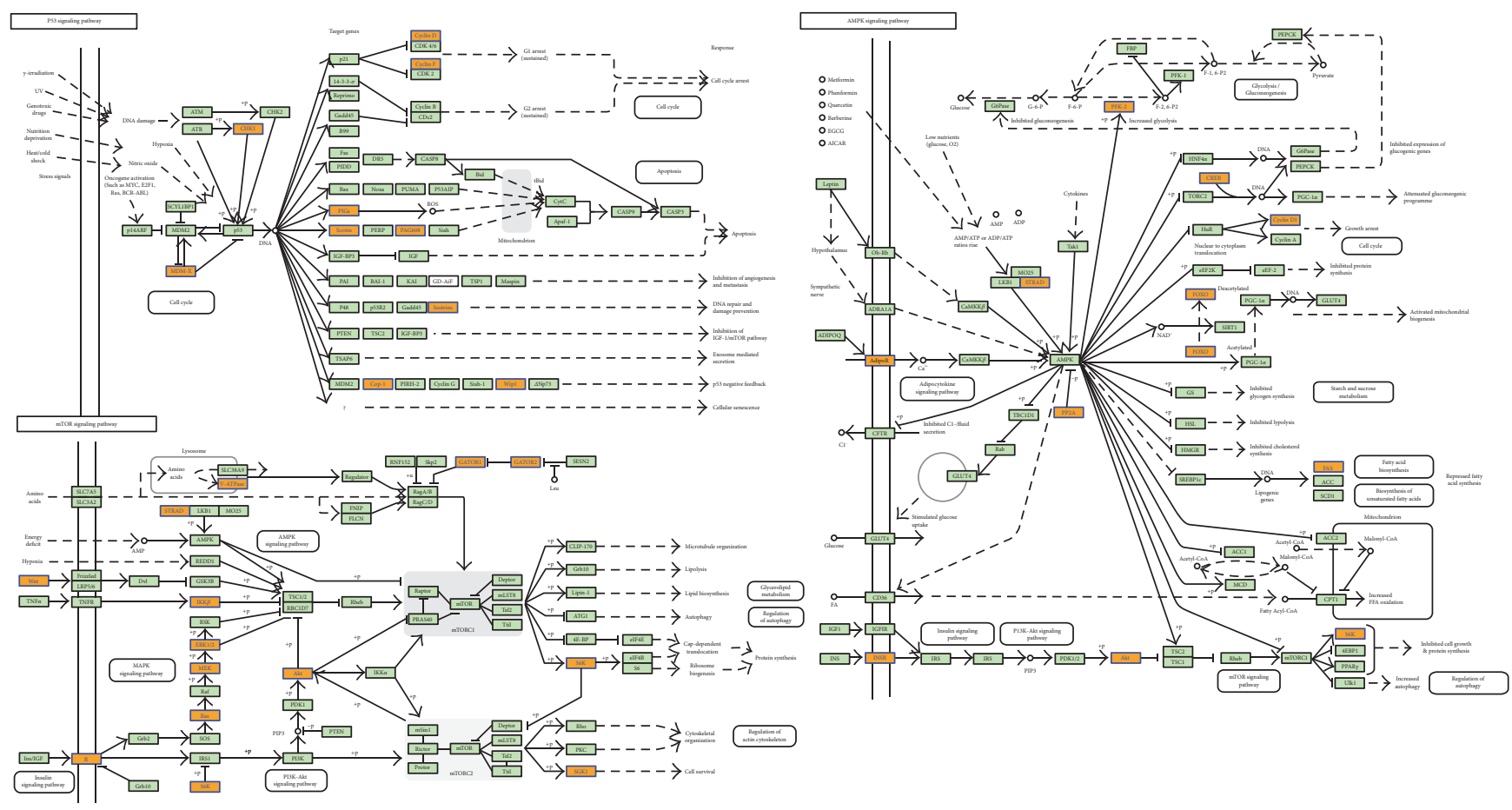

FIgURE 6: KEGG pathways of p53, mTOR, and AMPK.

The expression of miR-638 is known to be elevated in renal tubulointerstitial injury. In renal biopsies of patients with lupus nephritis, the expression of miR-638 was shown to be decreased in the glomeruli. However, tubulointerstitial miR-638 was increased and significantly correlated with proteinuria and the disease activity score [38]. In contrast, miR-133b expression was shown to be decreased in patients with polycystic kidney disease [39].

For the further explanation, we performed gene ontology (GO) function and KEGG pathway annotations for all ten miRNAs. For miR-877-3p, we found that the biological processes included "cell death," "epidermal growth factor receptor signaling pathway," and "response to hypoxia," and the KEGG pathways enriched included "p53 signaling pathway," "FoxO signaling pathway," and "Fatty acid biosynthesis and metabolism." Many studies have shown that these biological processes and pathways were closely related to DKD. Chronic hypoxia of the tubulointerstitium has been recognized as an important early event in diabetes mellitus [40]. Therefore, miR-877-3p might be involved in oxidative stress and sympathetic denervation of the kidney due to autonomic neuropathy, causing tubular apoptosis. Kato et al. had reported that Akt/FoxO pathway regulation might be a novel mechanism by which TGF beta induces unopposed MC survival and oxidant stress during early DKD [41]. These results suggested that miR-877-3p might increase FoxO3a phosphorylation and transcriptional inactivation via $\mathrm{PI} 3 \mathrm{~K} / \mathrm{Akt}$, thus accelerating renal disease.

The p53 (apoptosis-induced nuclear transcription factor), mammalian target of rapamycin (mTOR), and AMPactivated protein kinase (AMPK) pathways are known to play important roles in DKD. In a KEGG analysis, the predicted target genes of miRNAs were found to be related to p53,
mTOR, AMPK, malignancies, neurotrophic factors, and other signaling pathways. These results suggested that these miRNAs might also promote the expression of PIGs through the 553 pathway, thus activating the ROS system. Based on the results of the KEGG pathway analyses, we speculated that the elevated expression of miRNAs such as miR$362-3 p$ could mediate the activation of the Akt/mTOR pathway in diabetic podocytes. AMPK activation depends on the sestrin 2 protein (SESN2). Based on the predicted target genes, miR-15a-5p could target the regulation of SESN1, as SESN1/AMPK also has the potential to prevent fibrosis of diabetes mellitus function.

The miRNAs identified in our study might play important roles in the pathogenesis and progression of DKD, as evidenced by the high stability of UExo-derived miRNAs, compared to the free miRNAs in body fluids. A single miRNA can regulate multiple target genes, and multiple miRNAs can target a single gene. This was merely a crosssectional study, and basic experimental studies and cohort studies are still needed. Recently, ultrasound-microbubblemediated gene transfer has been applied to treat renal, peritoneal, hepatic, and other fibrosis conditions [42]. The microbubble technology has been used to knockdown miR-21 expression in $\mathrm{db} / \mathrm{db}$ mice, which alleviated microalbuminuria, renal fibrosis, and inflammation [43].

\section{Conclusions}

Although single miRNAs could be used as sensitive biomarkers for the diagnosis of DKD, they might exhibit poor specificity, as many miRNAs have regulatory roles in both DKD and non-DKD. Many studies have examined UExo-derived miRNAs using the microarray approach; we 
identified a number of new DKD-related miRNAs such as miR-362-3p, miR-877-3p, miR-150-5p, and miR-15a-5p, which might be novel candidate biomarkers for incipient DKD, especially miR-877-3p. Using multiple miRNAs to diagnose DKD could improve the specificity. By assessing the effect of each miRNA on DKD, we can weigh the relevant miRNAs using a formula to cover all the differentially expressed miRNAs to accurately assess the risk of DKD, thus contributing to early diagnosis. In general, UExo-derived miRNAs might be suitable biomarkers for detecting DKD, as well as potential therapeutic targets.

\section{Conflicts of Interest}

The authors declare that there is no conflict of interest regarding the publication of this article.

\section{Acknowledgments}

This work was supported by research grants from the National Natural Science Foundation of China (Grant no. 81570724) and the Natural Science Foundation of Guangdong (Grant no. 2014A030313290).

\section{References}

[1] KDOQI, "Clinical practice guidelines and clinical practice recommendations for anemia in chronic kidney disease," American Journal of Kidney Diseases, vol. 47, pp. S11-S145, 2006.

[2] B. Lu, W. Gong, Z. Yang et al., "An evaluation of the diabetic kidney disease definition in Chinese patients diagnosed with type 2 diabetes mellitus," The Journal of International Medical Research, vol. 37, pp. 1493-1500, 2009.

[3] L. F. Fried and J. Lewis, "Rebuttal of the pro view: albuminuria is an appropriate therapeutic target in patients with CKD," Clinical Journal of the American Society of Nephrology, vol. 10, pp. 1095-1098, 2015.

[4] R. W. Carthew and E. J. Sontheimer, "Origins and mechanisms of miRNAs and siRNAs," Cell, vol. 136, pp. 642-655, 2009.

[5] F. P. Schena, G. Serino, and F. Sallustio, "MicroRNAs in kidney diseases: new promising biomarkers for diagnosis and monitoring," Nephrology, Dialysis, Transplantation, vol. 29, pp. 755-763, 2014.

[6] K. Chandrasekaran, D. S. Karolina, S. Sepramaniam et al., "Role of microRNAs in kidney homeostasis and disease," Kidney International, vol. 81, pp. 617-627, 2012.

[7] J. Osipova, D. C. Fischer, S. Dangwal et al., "Diabetes-associated microRNAs in pediatric patients with type 1 diabetes mellitus: a cross-sectional cohort study," The Journal of Clinical Endocrinology and Metabolism, vol. 99, pp. E1661-E1665, 2014.

[8] G. Wang, B. C. Kwan, F. M. Lai, K. M. Chow, L. P. Kam-Tao, and C. C. Szeto, "Expression of microRNAs in the urinary sediment of patients with IgA nephropathy," Disease Markers, vol. 28, pp. 79-86, 2010.

[9] Y. Luo, C. Wang, X. Chen et al., "Increased serum and urinary microRNAs in children with idiopathic nephrotic syndrome," Clinical Chemistry, vol. 59, pp. 658-666, 2013.
[10] G. Wang, L. S. Tam, E. K. Li et al., "Serum and urinary free microRNA level in patients with systemic lupus erythematosus," Lupus, vol. 20, pp. 493-500, 2011.

[11] C. Argyropoulos, K. Wang, J. Bernardo et al., "Urinary microRNA profiling predicts the development of microalbuminuria in patients with type 1 diabetes," Journal of Clinical Medicine, vol. 4, pp. 1498-1517, 2015.

[12] J. A. Weber, D. H. Baxter, S. Zhang et al., "The microRNA spectrum in 12 body fluids," Clinical Chemistry, vol. 56, pp. 1733-1741, 2010.

[13] A. K. Ludwig and B. Giebel, "Exosomes: small vesicles participating in intercellular communication," The International Journal of Biochemistry \& Cell Biology, vol. 44, pp. 11-15, 2012.

[14] C. S. Neal, M. Z. Michael, L. K. Pimlott, T. Y. Yong, J. Y. $\mathrm{Li}$, and J. M. Gleadle, "Circulating microRNA expression is reduced in chronic kidney disease," Nephrology, Dialysis, Transplantation, vol. 26, pp. 3794-3802, 2011.

[15] A. Ramezani, J. M. Devaney, S. Cohen et al., "Circulating and urinary microRNA profile in focal segmental glomerulosclerosis: a pilot study," European Journal of Clinical Investigation, vol. 45, pp. 394-404, 2015.

[16] F. Barutta, M. Tricarico, A. Corbelli et al., "Urinary exosomal microRNAs in incipient diabetic nephropathy," PLoS One, vol. 8, article e73798, 2013.

[17] B. Wang, M. Herman-Edelstein, P. Koh et al., "E-cadherin expression is regulated by $\mathrm{miR}-192 / 215$ by a mechanism that is independent of the profibrotic effects of transforming growth factor- $\beta$," Diabetes, vol. 59, pp. 1794-1802, 2010.

[18] C. Argyropoulos, K. Wang, S. Mcclarty et al., "Urinary microRNA profiling in the nephropathy of type 1 diabetes," PLoS One, vol. 8, article e54662, 2013.

[19] D. Delić, C. Eisele, R. Schmid et al., "Urinary Exosomal miRNA signature in type II diabetic nephropathy patients," PLoS One, vol. 11, article e150154, 2016.

[20] Y. Jia, M. Guan, Z. Zheng et al., "miRNAs in urine extracellular vesicles as predictors of early-stage diabetic nephropathy," Journal of Diabetes Research, vol. 2016, Article ID 7932765, 10 pages, 2016.

[21] M. C. Riella, "Kidney disease: Improving global outcomes (KDIGO) CKD work group. KDIGO 2012 clinical practice guideline for the evaluation and management of chronic kidney disease," Kidney International Supplements 3.1, pp. 1150, 2013.

[22] R. Khurana, G. Ranches, S. Schafferer et al., "Identification of urinary exosomal noncoding RNAs as novel biomarkers in chronic kidney disease," RNA, vol. 23, no. 2, pp. 142-152, 2017.

[23] I. Z. Ben-Dov, T. Muthukumar, P. Morozov, F. B. Mueller, T. Tuschl, and M. Suthanthiran, "MicroRNA sequence profiles of human kidney allografts with or without tubulointerstitial fibrosis," Transplantation, vol. 94, pp. 1086-1094, 2012.

[24] A. H. Van Craenenbroeck, E. M. Van Craenenbroeck, A. K. Van et al., "Impaired vascular function contributes to exercise intolerance in chronic kidney disease," Nephrology, dialysis, transplantation: official publication of the European Dialysis and Transplant Association - European Renal Association, vol. 31, no. 12, pp. 2064-2072, 2016.

[25] T. Pisitkun, R. F. Shen, and M. A. Knepper, "Identification and proteomic profiling of exosomes in human urine," Proceedings 
of the National Academy of Sciences of the United States of America, vol. 101, p. 13368, 2004.

[26] B. S. Zitkus, "American Diabetes Association Standards of Medical Care in Diabetes," Nurse Practitioner, vol. 30, pp. S12-S54, 2014.

[27] C. Wang, S. Gu, H. Cao et al., "miR-877-3p targets Smad7 and is associated with myofibroblast differentiation and bleomycin-induced lung fibrosis," SCI REP-UK, vol. 6, article 30122, 2016.

[28] E. Flowers, M. Gadgil, B. E. Aouizerat, and A. M. Kanaya, "Circulating microRNAs associated with glycemic impairment and progression in Asian Indians," Biomarker Research, vol. 3, pp. 1-8, 2015.

[29] J. Muralidharan, A. Ramezani, M. Hubal et al., "Extracellular microRNA signature in chronic kidney disease," American Journal of Physiology - Renal Physiology, vol. 312, no. 6, pp. F982-F991, 2016.

[30] X. Meng, D. J. Nikolic-Paterson, and H. Y. Lan, “TGF- $\beta$ : the master regulator of fibrosis," Nature Reviews. Nephrology, vol. 12, no. 6, pp. 325-338, 2016.

[31] B. Wang, J. C. Jha, S. Hagiwara et al., "Transforming growth factor-beta1-mediated renal fibrosis is dependent on the regulation of transforming growth factor receptor 1 expression by let-7b," Kidney International, vol. 85, pp. 352-361, 2014.

[32] J. Wang, Y. Gao, M. Ma et al., "Effect of miR-21 on renal fibrosis by regulating MMP-9 and TIMP1 in kk-ay diabetic nephropathy mice," Cell Biochemistry and Biophysics, vol. 67, pp. 537-546, 2013.

[33] X. J. Liu, Q. Hong, Z. Wang, Y. Y. Yu, X. Zou, and L. H. Xu, "MicroRNA21 promotes interstitial fibrosis via targeting DDAH1: a potential role in renal fibrosis," Molecular and Cellular Biochemistry, vol. 411, pp. 181-189, 2016.

[34] H. Bao, S. Hu, C. Zhang et al., "Inhibition of miRNA-21 prevents fibrogenic activation in podocytes and tubular cells in IgA nephropathy," Biochemical and Biophysical Research Communications, vol. 444, pp. 455-460, 2014.

[35] P. Du, B. Fan, H. Han et al., "NOD2 promotes renal injury by exacerbating inflammation and podocyte insulin resistance in diabetic nephropathy," Kidney International, vol. 84, p. 265, 2013.

[36] H. Zhou, S. A. Hasni, P. Perez et al., "miR-150 promotes renal fibrosis in lupus nephritis by downregulating SOCS1," Journal of the American Society of Nephrology, vol. 24, pp. 1073-1087, 2013.

[37] S. Eissa, M. Matboli, and M. M. Bekhet, "Clinical verification of a novel urinary microRNA panal: $133 b,-342$ and -30 as biomarkers for diabetic nephropathy identified by bioinformatics analysis," Biomedicine \& pharmacotherapy = Biomedecine \& pharmacotherapie, vol. 83, pp. 92-99, 2016.

[38] J. Lu, B. C. Kwan, F. M. Lai et al., "Glomerular and tubulointerstitial miR-638, miR-198 and miR-146a expression in lupus nephritis," Nephrology, vol. 17, pp. 346-351, 2012.

[39] I. Z. Bendov, Y. C. Tan, P. Morozov et al., "Urine microRNA as potential biomarkers of autosomal dominant polycystic kidney disease progression: description of miRNA profiles at baseline," PLoS One, vol. 9, article e86856, 2013.

[40] D. K. Singh, P. Winocour, and K. Farrington, "Mechanisms of disease: the hypoxic tubular hypothesis of diabetic nephropathy," Nature Clinical Practice. Nephrology, vol. 4, pp. 216-226, 2008.
[41] M. Kato, H. Yuan, Z. G. Xu et al., "Role of the Akt/FoxO3a pathway in TGF-beta1-mediated mesangial cell dysfunction: a novel mechanism related to diabetic kidney disease," Journal of the American Society of Nephrology, vol. 17, pp. 3325-3335, 2007.

[42] Z. Z. Jiang, G. Y. Xia, and Z. K. Xiong, "Application of ultrasound-microbubble-mediated gene transfer in the treatment of tissue fibrosis," Chinese Journal of Medical Imaging Technology, vol. 26, no. 5, pp. 983-985, 2010.

[43] X. Zhong, A. C. Chung, H. Y. Chen et al., "miR-21 is a key therapeutic target for renal injury in a mouse model of type 2 diabetes," Diabetologia, vol. 56, p. 663, 2013. 


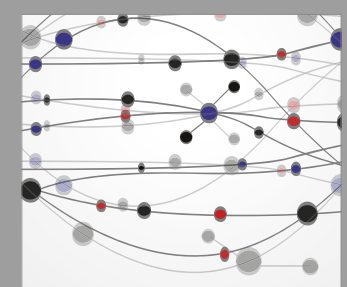

The Scientific World Journal
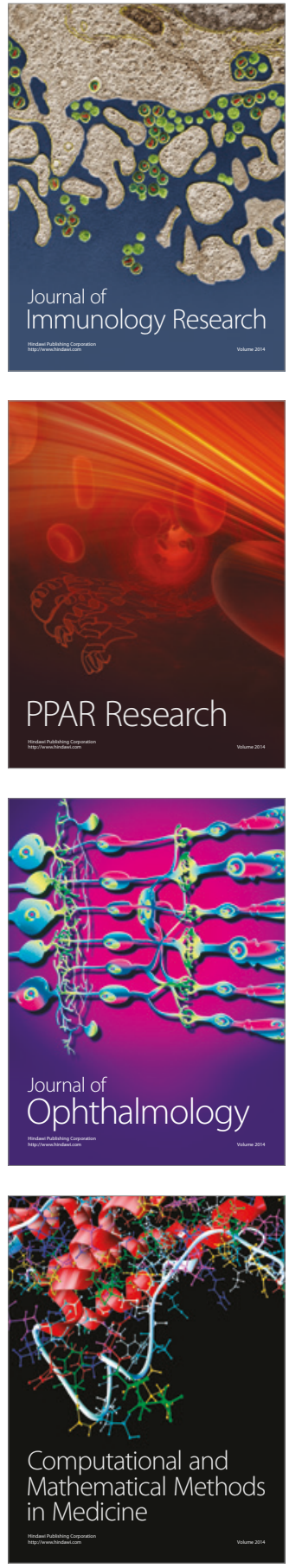

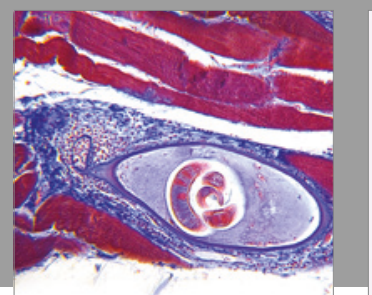

Gastroenterology Research and Practice
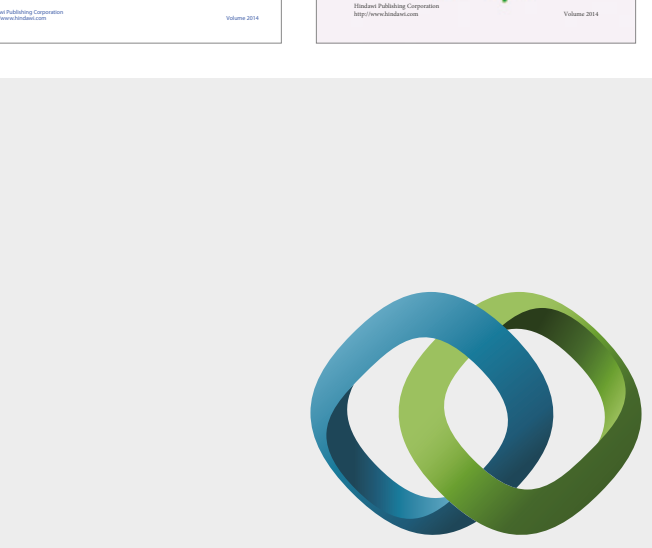

\section{Hindawi}

Submit your manuscripts at

https://www.hindawi.com
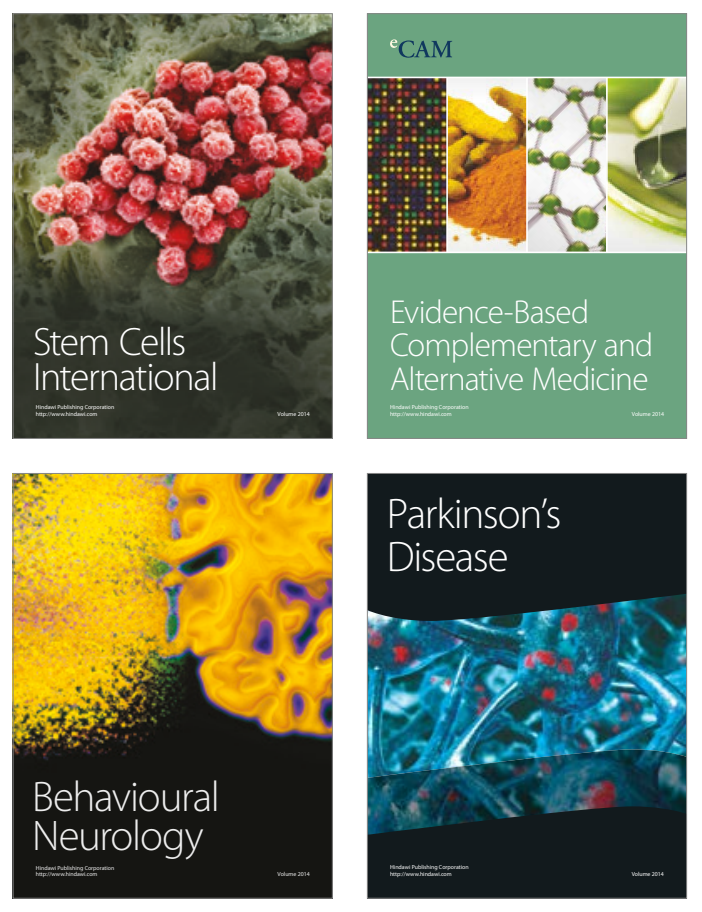
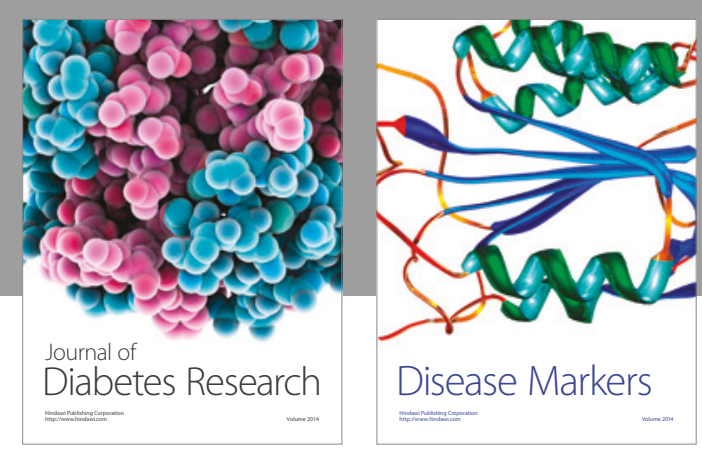

Disease Markers
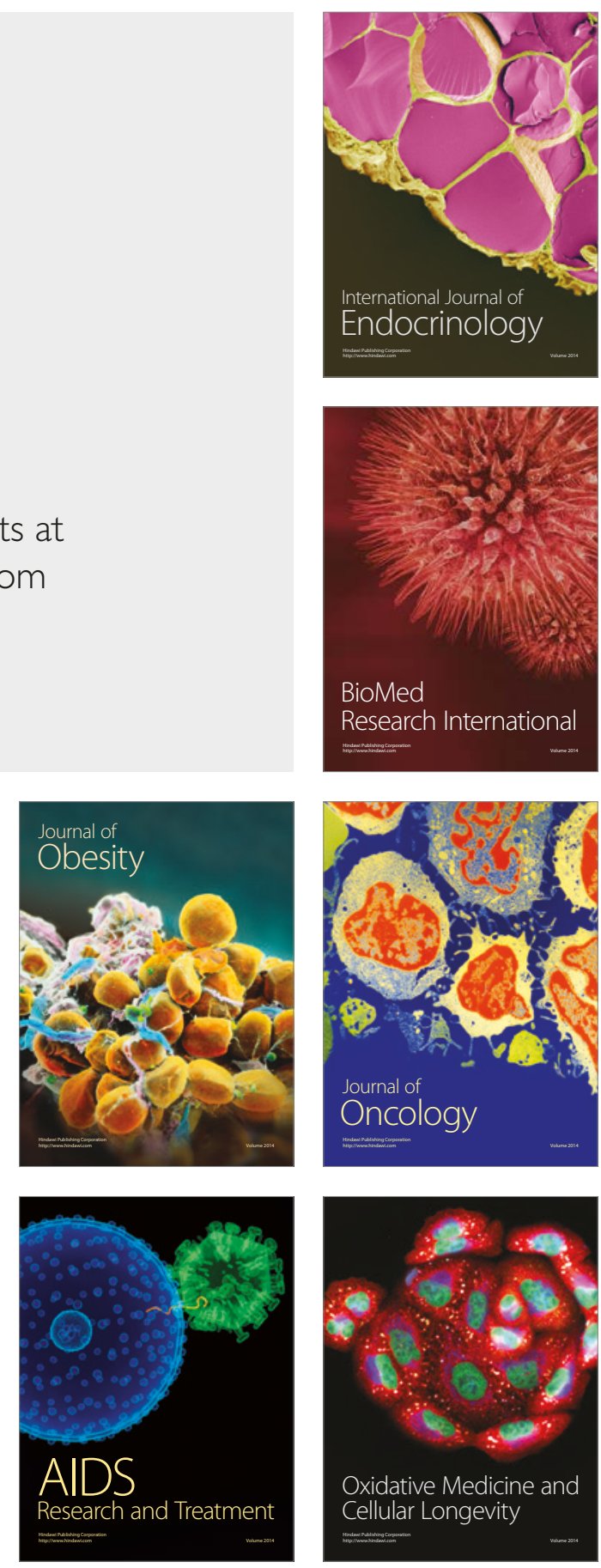